\title{
Optimal Switching Strategy between Admission Control and Pricing Control Policies with Two Types of Customers and Search Costs
}

\author{
Jae-Dong Son \\ Department of Industrial and Information Systems Engineering, Soongsil University, 511 Sangdo-dong, Dongjak-ku, \\ Seoul 156-743, Republic of Korea \\ Correspondence should be addressed to Jae-Dong Son; son88@ssu.ac.kr
}

Received 19 September 2014; Accepted 9 March 2015

Academic Editor: Viliam Makis

Copyright (C) 2015 Jae-Dong Son. This is an open access article distributed under the Creative Commons Attribution License, which permits unrestricted use, distribution, and reproduction in any medium, provided the original work is properly cited.

\begin{abstract}
This paper presents a switching strategy between the admission control and the pricing control policies in a queueing system with two types of customers. For an arriving first-type customer, the decision maker has an option on which policy to choose between the two control policies; that is, one determines whether or not to admit the customer's request for the service (admission control) or decides a price of the customer's request and offers it to the customer (pricing control). The second-type customers are only served when no first-type customers are present in the system in order to prevent the system from being idle. This would yield an extra income, which we refer to as the sideline profit. The so-called search cost, which is a cost paid to search for customers, creates the search option on whether to continue the search or not. We clarify the properties of the optimal switching strategy as well as the optimal search policy in relation to the sideline profit in order to maximize the total expected net profit. In particular, we show that when the sideline profit is sufficiently large, the two optimal switching thresholds exist with respect to the number of first-type customers in the system.
\end{abstract}

\section{Introduction}

Both the admission and pricing control policies have been widely investigated to improve the performance of queueing system in the telecommunications and manufacturing industries [1-6]. In the admission control [7-10], an arriving customer proposes a price for his request, and the decision maker decides the admission merely based upon the proposed price of the customer. In general, it is assumed that the customer has one's own maximum permissible offering price, which is also referred to as the reservation price, and the stronger the customers' desire to be served is, the closer to the reservation price may be proposed. On the other hand, in the pricing control [11-14], the decision maker suggests a price to an arriving customer who has a service request. The customer then makes a service request to the company if and only if this suggested price is lower than or equal to the reservation price. Hence the decision maker should determine the offering price to maximize the expected profit.
Most of the prior researches have adopted exactly one of the two forementioned polices in accordance with their model's characteristics. However, in what follows, both types of policies were considered: first, Yoon and Lewis [15] formulated and analyzed the problems involving both admission and pricing control policies but as distinct entities. Furthermore, Gans and Savin [16] considered a rental firm with two types of customers where one is controlled by the admission policy and the other by the pricing policy. Hew and White [17] integrated a call admission and dynamic pricing problem with handoffs and price-affected arrivals. In their formulation, the former arrivals are controlled by only the admission policy, while the latter ones are sequentially controlled by both policies. Son [18] gave separate formulations of the admission and the pricing control problems, yet showing that both problems can be analyzed within an identical framework.

So far, whether or not we consider these two policies separately or within an identical framework, the switching 
strategy between the two has not been introduced. In reality, a system manager is not restricted to use only one policy to control the system capacity. One may switch the control policy from one type to the other as long as a higher profit is expected. As the competition gets tougher in business nowadays, companies are open to new control strategies that may have been considered unrealistic up to now if there are chances of yielding higher profit.

Motivated by these observations, we propose a switching strategy between admission control and pricing control policies. The research on a basic switching strategy in an $\mathrm{m} / \mathrm{m} / 1 / \mathrm{k}$ queueing system, introduced in [19], showed the existence of switching thresholds in terms of the number of customers in the system. It demonstrated that by employing the switching strategy, the maximum total expected profit can be improved significantly as high as $8.7 \%$.

In this paper, we apply this switching strategy to a service providing company with two types of customers where the priority is given to the first type over the second. For example, let us consider a manpower company with two types of customers: the first-type customers request a personnel training, and the second type ask for a temporary staffing. The crucial performance of the manpower company is characterized by placing greater emphasis on providing the personnel training service. Indeed, since their topmost priority is to serve the first-type customers, the company would devote itself to meet their needs. Thus, the second-type customers are served as a sideline to avoid idling the server when no first-type customers are present in the system. This would yield an extra income, which we will refer to as the sideline profit.

We also introduce in the paper the so-called search cost; that is, the cost a company would spend in order to find customers. The search cost has been introduced in the conventional optimal stopping problem $([20-22])$ but not in the conventional control problems like the one discussed in [19]. By introducing the search cost in the system, it would eventually create a search option on whether or not to conduct the search. Therefore, the objective of this paper is to examine the structure of an optimal switching strategy as well as an optimal search policy in relation to the sideline profit in order to maximize the total expected net profit over an infinite planning horizon.

The paper is organized as follows: in Section 2, we present a switching model between the admission and pricing control policies with the set of optimality equations of the model. In Section 3, we transform these optimality equations to produce the optimal switching and search strategies. In Section 4, we examine the properties of the optimal switching policies obtained in the preceding section and discuss some important aspects of the model via numerical studies in Section 5. Finally, a conclusion is given in Section 6.

\section{Model Formulation}

A discrete-time queueing model is defined on the following seven assumptions.

(1) The model in the paper is defined as a single server with the system capacity $k$ with two types of customers.
(2) The first-type customer appears with the probability $\lambda(0<\lambda \leq 1)$ only after the search $\operatorname{cost} c \geq 0$ has been paid at the previous point in time.

(3) The service for the admitted request is completed with the probability $q(0<q<1)$ at the next point in time.

(4) Since the first-type customers are taken prior to the second-type customers, the latter are served only when no first-type customers are available. By $\rho(\geq 0)$ let us denote the sideline profit obtained by serving the second-type customers for one unit time. Henceforth, the term "customer" will refer to a first-type customer.

(5) The customers arriving sequentially are assumed to have their reservation prices, $\xi_{1}, \xi_{2}, \ldots$, which are i.i.d random variables determined by a known distribution function $F_{\xi}(x)$ with the expectation value $\mu_{\xi}$, and the density function is defined as follows:

$$
\begin{gathered}
f_{\xi}(x)>0, \quad \text { if } a \leq x \leq b, \\
f_{\xi}(x)=0, \quad \text { otherwise, }
\end{gathered}
$$

where $a$ and $b(0<a<b<\infty)$ are certain given numbers.

(6) If the admission control policy is adopted for an arriving customer with the reservation price $\xi$, the decision maker then has to determine whether or not to admit the customer based on the price $w=\alpha \xi$, where $\alpha \in[0,1]$ is a ratio which denotes the customer's degree of desirability for the service; that is, the greater the customer's desirability is, the closer $\alpha$ is to 1 . These ratios $\alpha_{1}, \alpha_{2}, \ldots$ are i.i.d random variables from a known distribution function $F_{\alpha}(x)$ with the expectation $\mu_{\alpha}$. Therefore, it is clear that $\mu_{w}=\mu_{\alpha} \mu_{\xi}$. Now the distribution function of $w$ will be

$$
\begin{aligned}
F_{w}(x) & =\operatorname{Pr}(w \leq x)=\operatorname{Pr}(\alpha \xi \leq x)=\operatorname{Pr}\left(\xi \leq \frac{x}{\alpha}\right) \\
& =\int_{0}^{\infty} F_{\xi}\left(\frac{x}{\alpha}\right) f_{\alpha}(\alpha) d \alpha=E_{\alpha}\left[F_{\xi}\left(\frac{x}{\alpha}\right)\right],
\end{aligned}
$$

and the density function becomes

$$
f_{w}(x)=E_{\alpha}\left[\frac{1}{\alpha} f_{\xi}\left(\frac{x}{\alpha}\right)\right] .
$$

(7) If the pricing control policy is adopted, the decision maker then proposes a price $z$ to the customer's request and the customer makes a service request if and only if the proposed price $z$ is less than or equal to his own reservation price $\xi$. Accordingly, the probability that the customer requests the service will be $p(z)=\operatorname{Pr}(z \leq \xi)$.

Let us denote by $i$ the number of customers in the system, and let $V(i)$ represent the maximum expected net profit in the current state $i$. In such case, by using the Markovian decision 
process we can describe the optimality equations of the model as follows:

$$
\begin{gathered}
V(0)=\max \left\{\mathrm{C}: \beta\left(\lambda \operatorname { m a x } \left\{E_{w}[\max \{w+V(1), V(0)\}],\right.\right.\right. \\
\max _{z}\{p(z)(z+V(1)) \\
+(1-p(z)) V(0)\}\} \\
+(1-\lambda) V(0))-c, \\
\mathrm{~K}: \beta V(0)\}+\rho,
\end{gathered}
$$

$V(i)$

$$
\begin{gathered}
=\max \{\mathrm{C}: \beta(1-q) \\
\cdot\left(\left(\lambda \operatorname { m a x } \left\{E_{w}[\max \{w+V(i+1), V(i)\}],\right.\right.\right. \\
\max _{z}\{p(z)(z+V(i+1)) \\
+(1-p(z)) V(i)\}\} \\
\left.+(1-\lambda) V(i)) I_{(1 \leq i<k)}+V(i) I_{(i=k)}\right) \\
+\beta q\left(\lambda \operatorname { m a x } \left\{E_{w}[\max \{w+V(i), V(i-1)\}],\right.\right. \\
\max _{z}\{p(z)(z+V(i)) \\
+(1-p(z)) V(i-1)\}\} \\
+(1-\lambda) V(i-1))-c, \\
\mathrm{~K}: \beta(1-q) V(i)+\beta q V(i-1)\}
\end{gathered}
$$$$
\text { for } 1 \leq i \leq k
$$

where $\beta$ and $I_{(\cdot)}$ represent the discount factor and the indicator function, respectively. The letters $\mathrm{C}$ and $\mathrm{K}$ denote the decision of conducting the search and skipping the search, respectively.

To explain this model in more details, suppose that a customer appears with the probability $\lambda$ in the state $i$ having paid the search cost $c$. When adopting the admission control policy, in the case that the customer proposes the price $w$ for the service and the company admits it, the profit $w$ would be obtained and the state would increase to $i+1$; otherwise, the state would remain as $i$. When the pricing control policy is employed instead, as we mentioned before we first assume that the decision maker offers the price $z$ to an arriving customer in state $i$. If the customer requests his service with probability $p(z)$, the company obtains the profit $z$ and the state becomes $i+1$; otherwise, the state remains as $i$. Hence, the decision maker should offer a price $z$ that pertains to the maximization of $\{p(z)(z+V(i+1))+(1-p(z)) V(i)\}$. Note that when $i=0$, the service for a second-type customer will be provided and the reward $\rho$ is obtained for one unit time. Furthermore, when $i=k$, if the current service is not yet completed with the probability $(1-q)$, due to the service capacity an arriving customer's request can not be met and so the state remains as $i$.

\section{Transformation of the Optimality Equations}

In this section, we transform the optimality equations described in the preceding section and present the optimal switching and search policies. Let us begin by defining

$$
h_{i}=V(i)-V(i+1), \quad 0 \leq i \leq k-1 .
$$

For concise expression and for the convenience of model analysis, let us further define the following functions:

$$
\begin{aligned}
T_{p}(x) & =\max _{z} p(z)(z-x) \\
T_{w}(x) & =E_{w}[\max \{w-x, 0\}] \\
J(x) & =T_{w}(x)-T_{p}(x) \\
K(x) & =\max \left\{T_{w}(x), T_{p}(x)\right\}=\max \{J(x), 0\}+T_{p}(x)
\end{aligned}
$$

Moreover, we will denote the value $z$ which maximizes $p(z)(z-x)$ by $z(x)$ in (7). Corresponding to (8), we will also define

$$
b^{o}=\sup \left\{x \mid T_{w}(x)>0\right\} .
$$

Since the expectation of immediate reward at any point in time is finite, by using the conventional method outlined in a Markovian decision process [23], we can easily verify that $V(i) \leq M /(1-\beta)$ for a sufficiently large $M>0$; that is, $V(i)$ is finite. Hence, we can see that the system of (4) and (5) has a unique solution, regardless of the details of the optimal decisions. Now, the terms $\max \{w+V(i+1), V(i)\}$ and $\max _{z}\{p(z)(z+V(i+1))+(1-p(z)) V(i)\}$ can be represented as $\max \{w+V(i+1)-V(i), 0\}+V(i)$ and $\max _{z} p(z)(z+V(i+1)-$ $V(i))+V(i)$, respectively, that is, $\max \left\{w-h_{i}, 0\right\}+V(i)$ and 
$\max _{z} p(z)\left(z-h_{i}\right)+V(i)$ via the definition in (6). Hence, (4) and (5) now become

$$
\begin{aligned}
V(0)= & \beta V(0) \\
& +\max \left\{\beta \lambda \operatorname { m a x } \left\{E_{w}\left[\max \left\{w-h_{0}, 0\right\}\right],\right.\right. \\
& \left.\left.\max _{z} p(z)\left(z-h_{0}\right)\right\}-c, 0\right\}+\rho,
\end{aligned}
$$

$V(i)$

$$
\begin{gathered}
=\beta(1-q) V(i)+\beta q V(i-1) \\
+\beta \lambda \max \left\{( 1 - q ) \operatorname { m a x } \left\{E_{w}\left[\max \left\{w-h_{i}, 0\right\}\right],\right.\right. \\
\left.\max _{z} p(z)\left(z-h_{i}\right)\right\} I_{(1 \leq i<k)} \\
+q \max \left\{E_{w}\left[\max \left\{w-h_{i-1}, 0\right\}\right],\right. \\
\left.\left.\max _{z} p(z)\left(z-h_{i-1}\right)\right\}-c, 0\right\}, \\
1 \leq i \leq k .
\end{gathered}
$$

Then using (7) to (10), we rearrange these equations as follows:

$$
\begin{aligned}
& V(0)=\frac{\left(\max \left\{\beta \lambda\left(\max \left\{J\left(h_{0}\right), 0\right\}+T_{p}\left(h_{0}\right)\right)-c, 0\right\}+\rho\right)}{(1-\beta)}, \\
& \begin{array}{r}
V(i) \\
=\gamma \beta q V(i-1) \\
+\gamma \max \left\{\beta(1-q) \lambda\left(\max \left\{J\left(h_{i}\right), 0\right\}+T_{p}\left(h_{i}\right)\right) I_{(1 \leq i<k)}\right. \\
\left.\quad+\beta q \lambda\left(\max \left\{J\left(h_{i-1}\right), 0\right\}+T_{p}\left(h_{i-1}\right)\right)-c, 0\right\}, \\
1 \leq i \leq k,
\end{array}
\end{aligned}
$$

where $\gamma=(1-\beta(1-q))^{-1}>1$, which lead to the following optimality equations:

$$
\begin{gathered}
V(0)=\frac{\left(\max \left\{Q_{0}, 0\right\}+\rho\right)}{(1-\beta)}, \\
V(i)=\gamma \beta q V(i-1)+\gamma \max \left\{Q_{i}, 0\right\}, \quad 1 \leq i \leq k,
\end{gathered}
$$

where $Q_{0}=\beta \lambda K\left(h_{0}\right)-c$ and $Q_{i}=\beta(1-q) \lambda K\left(h_{i}\right) I_{(1 \leq i<k)}+$ $\beta q \lambda K\left(h_{i-1}\right)-c$ for $1 \leq i \leq k$.

In what comes below, we derive some equations related to $h_{i}$. By setting $i=0$ in (6), we obtain $h_{0}=V(0)-V(1)$, and replacing $V(1)$ by (15) with $i=1$ produces $h_{0}=(1-$ $\gamma \beta q) V(0)-\gamma \max \left\{Q_{1}, 0\right\}$. Since $1-\gamma \beta q=\gamma(1-\beta)$, this can be rewritten as $h_{0}=\gamma(1-\beta) V(0)-\gamma \max \left\{Q_{1}, 0\right\}$. Combining this with (14) now leads to

$$
h_{0}=\gamma \max \left\{Q_{0}, 0\right\}-\gamma \max \left\{Q_{1}, 0\right\}+\gamma \rho .
$$

In a similar way, for $1 \leq i \leq k-1$, we obtain the following equation:

$$
h_{i}=\gamma \beta q h_{i-1}+\gamma \max \left\{Q_{i}, 0\right\}-\gamma \max \left\{Q_{i+1}, 0\right\}, \quad 1 \leq i<k .
$$

Based upon our discussions so far, we are now ready to describe the optimal policies with respect to the switching strategy and the search option for a given state as follows.

\section{Optimal Policies}

(a) Optimal Switching Strategies. For $0 \leq i<k$, we have the following.

(1) If $J\left(h_{i}\right)>0$, adopt the admission control policy (see (13)). In this case, if the proposed price $w$ of an arriving customer is greater than $h_{i}$, then it is optimal to admit the customer is optimal in state $i$; otherwise rejection is optimal (see (12)).

(2) If $J\left(h_{i}\right) \leq 0$, adopt the pricing control policy (see (13)). In this case, the optimal price to offer to an arriving customer is determined by $z\left(h_{i}\right)$ which maximizes $p(z)\left(z-h_{i}\right)$ in state $i$ (see (12)).

(b) Optimal Search Strategies. For $0 \leq i \leq k$, it is optimal to conduct the search if $Q_{i}>0$, and to skip it otherwise (see (14) and (15)).

\section{Results}

In what follows, we examine the structure of the optimal policies described above and consider their implications.

Theorem 1. If $\beta \lambda K(0) \leq c$, then $Q_{i} \leq 0$ for $0 \leq i \leq k$.

Proof. Assume $\beta \lambda K(0) \leq c$. Then from Lemmas A.2 and A.l(b) we have $0 \geq \beta \lambda K(0)-c \geq \beta \lambda K\left(h_{0}\right)-c=Q_{0}, 0 \geq$ $\beta \lambda K(0)-c=(1-q)(\beta \lambda K(0)-c)+q(\beta \lambda K(0)-c) \geq(1-$ $q)\left(\beta \lambda K\left(h_{i}\right)-c\right)+q\left(\beta \lambda K\left(h_{i}-1\right)-c\right)=Q_{i}$ for $1 \leq i<k$, and $0 \geq \beta \lambda K(0)-c>\beta q \lambda K\left(h_{k-1}\right)-c=Q_{k}$.

This result indicates that when the search cost $c$ is sufficiently large as $c \geq \beta \lambda K(0)$, it is optimal not to conduct the search for customers; hence no first-type customers are present in the system. As a result the company will provide the service only for the second-type customers.

Therefore, we consider only the nontrivial case $\beta \lambda K(0)>$ $c$ in remainder of this section.

Theorem 2. Suppose $h_{i-1} \leq h_{i}$ for a given $i(1 \leq i<k)$. Then one has $Q_{j}>0$ for $j$ with $i \leq j<k$ and

(a) $h_{i-1} \leq h_{i} \leq \cdots \leq h_{n-1}<b$,

(b) $z\left(h_{i-1}\right) \leq z\left(h_{i}\right) \leq \cdots \leq z\left(h_{n-1}\right)<b$. 
Proof. (a) Let $h_{i-1}<h_{i}$ for a given $i(1 \leq i<k)$; hence $h_{i-1} \leq$ $h_{i}$. Then from the contrapositions of Lemma A.3 we get $Q_{i}>$ 0 ; accordingly, using Lemma A.1(b) we have

$$
\begin{aligned}
0<Q_{i} & =\beta(1-q) \lambda K\left(h_{i}\right)+\beta q \lambda K\left(h_{i-1}\right)-c \\
& \leq \beta(1-q) \lambda K\left(h_{i-1}\right)+\beta q \lambda K\left(h_{i-1}\right) \\
& =\beta \lambda K\left(h_{i-1}\right)-c
\end{aligned}
$$

which gives $K\left(h_{i-1}\right)>c / \beta \lambda \geq 0$, and hence $h_{i-1}<b$ due to Lemma A.1(c). Further, from (17) we have

$$
\begin{aligned}
h_{i}= & \gamma q \beta h_{i-1}+\gamma \beta(1-q) \lambda K\left(h_{i}\right)+\gamma \beta q \lambda K\left(h_{i-1}\right) \\
& -\gamma c-\gamma \max \left\{Q_{i+1}, 0\right\} \\
\leq & \gamma q \beta\left(h_{i-1}+\lambda K\left(h_{i-1}\right)\right)+\gamma \beta(1-q) \lambda K\left(h_{i}\right)-\gamma c .
\end{aligned}
$$

Assume $h_{i} \geq b$. Then the above inequality becomes $h_{i} \leq$ $\gamma q \beta\left(h_{i-1}+\lambda K\left(h_{i-1}\right)\right)-\gamma c$ due to $K\left(h_{i}\right)=0$ from Lemma A.1(c). Since $h_{i-1}<b$, using Lemma A.1(d), we have $h_{i} \leq \gamma q \beta(b+$ $K(b))-\gamma c=\gamma q \beta(b-0)-\gamma c \leq \gamma q \beta b<b$ due to $\gamma q \beta<1$, which is a contradiction. Hence, it must be $h_{i-1}<(\leq) h_{i}<$ $b$. Noting this result and Lemma A.1(d), we arrange (19) as $h_{i} \leq \gamma q \beta h_{i}+\gamma \beta \lambda K\left(h_{i}\right)-\gamma c$, and this inequality becomes $(1-\gamma q \beta) h_{i} \leq \gamma \beta \lambda K\left(h_{i}\right)-\gamma c$. Since $h_{i-1} \geq 0$ from Lemma A.2, we have $h_{i}>0$ due to the assumption $h_{i-1}<(\leq) h_{i}$. From this and the fact that $1>\gamma q \beta$ we obtain $(1-\gamma q \beta) h_{i}>0 \cdots\left(1^{*}\right)$; hence $\gamma\left(\beta \lambda K\left(h_{i}\right)-c\right)>0 \cdots\left(2^{*}\right)$. Now, suppose $Q_{i+1} \leq 0$. Then Lemma A.3 and the above result give $h_{i+1} \leq h_{i}<b$. Moreover, from Lemma A.1(c) we have

$$
\begin{aligned}
0 & \geq Q_{i+1}=\beta(1-q) \lambda K\left(h_{i+1}\right)+\beta q \lambda K\left(h_{i}\right)-c \\
& \geq \beta(1-q) K\left(h_{i}\right)+\beta q \lambda K\left(h_{i}\right)-c \\
& =\beta \lambda K\left(h_{i}\right)-c,
\end{aligned}
$$

which contradicts $\left(2^{*}\right)$; thus $Q_{i+1}>0$. Because both $Q_{i}$ and $Q_{i+1}$ are positive, we can rewrite (17) as $h_{i}=\gamma \beta q\left(h_{i-1}+\right.$ $\left.\lambda K\left(h_{i-1}\right)\right)+\gamma \beta(1-2 q) K\left(h_{i}\right)-\gamma \beta(1-q) K\left(h_{i+1}\right)$. Noting the assumption $h_{i-1}<(\leq) h_{i}$, from Lemma A.1(d) we get

$$
\begin{aligned}
h_{i} \leq & \gamma \beta q\left(h_{i}+\lambda K\left(h_{i}\right)\right)+\gamma \beta(1-2 q) \lambda K\left(h_{i}\right) \\
& -\gamma \beta(1-q) K\left(h_{i+1}\right) \\
= & \gamma \beta q h_{i}+\gamma \beta(1-q)\left(K\left(h_{i}\right)-K\left(h_{i+1}\right)\right),
\end{aligned}
$$

which leads to $(1-\gamma \beta q) h_{i} \leq \gamma \beta(1-q)\left(K\left(h_{i}\right)-K\left(h_{i+1}\right)\right)$. Since $(1-\gamma q \beta) h_{i}>0$ from $\left(1^{*}\right)$, we get $K\left(h_{i+1}\right) \leq K\left(h_{i}\right)$, implying that $h_{i} \leq h_{i+1}$ due to Lemma A.1(b). Repeating the same procedure leads to the completion of the induction.

(b) It is immediate from the fact that $z(x)$ is increasing in $x([24])$.

From the above result we see that if $h_{i-1}<h_{i}$ for a given state $i(1 \leq i<k)$, then the optimal admission threshold $h_{j}$ and the optimal pricing $z\left(h_{j}\right)$ are given as increasing functions in the number of customers $j$ with $i \leq j<k$. Therefore, $h_{i}$ and $z\left(h_{i}\right)$ appear as one of the following functions. (1) Both $h_{i}$ and $z\left(h_{i}\right)$ are decreasing in $i$. (2) For some value $m>0$, both $h_{i}$ and $z\left(h_{i}\right)$ are decreasing in $i<m$ and increasing in $i \geq m$, which means that both $h_{i}$ and $z\left(h_{i}\right)$ are convex unimodal in $i$. (3) Both $h_{i}$ and $z\left(h_{i}\right)$ are increasing in $i$.

Theorem 3. If $Q_{i}>0$ for a given $i(0 \leq i<k)$, then $Q_{j}>0$ for $i \leq j<k$.

Proof. Let $Q_{i}>0$ for a given $i(1 \leq i<n)$. First, assume $h_{i-1}<h_{i}$. Then $Q_{i+1}>0$ from Theorem 2. Next, let $h_{i-1} \geq h_{i}$. Then since $K\left(h_{i-1}\right) \leq K\left(h_{i}\right)$ due to Lemma A.1(c), we get $0<$ $Q_{i}=\beta(1-q) \lambda K\left(h_{i}\right)+\beta q \lambda K\left(h_{i-1}\right)-c \leq \beta \lambda K\left(h_{i}\right)-c$. Suppose $Q_{i+1} \leq 0$. Then $h_{i} \geq h_{i+1}$ due to Lemma A.3. Noting $K(x)$ is convex on $(-\infty, \infty)$ from Lemma A.1(b), we have $\beta \lambda K((1-$ $\left.q) h_{i+1}+q h_{i}\right)-c \leq \beta \lambda\left((1-q) K\left(h_{i+1}\right)+q K\left(h_{i}\right)\right)-c \leq 0<$ $\beta \lambda K\left(h_{i}\right)-c$, implying $K\left((1-q) h_{i+1}+q h_{i}\right)<K\left(h_{i}\right)$, and hence $(1-q) h_{i+1}+q h_{i}>h_{i}$ due to Lemma A.1(b). Therefore, $h_{i}<h_{i+1}$ due to the assumption $q<1$, which is a contradiction. Thus, $Q_{i+1}>0$. We can complete the induction by repeating the same procedure. Now, if $Q_{0}>0$ and $Q_{1} \leq 0$, we get to a contradiction in quite the same way as above, so it must be that $Q_{1}>0$.

The above result states that if it is optimal to continue the search in a given state $i$, then it will be so in all states $j \geq i$. This means that, starting from the initial state 0 , if $Q_{0}>0$, then the optimal policy is to conduct the search in all states.

Theorem 4. Let $\rho=0$. If $\beta \lambda K(0)>c$, one has

(a) $Q_{i}>0$ for $0 \leq i<k$,

(b) $h_{i}$ and $z\left(h_{i}\right)$ are increasing in $i(0 \leq i<k)$.

Proof. (a) Since $V(i) \geq 0$ from (14) and (15), we have $V(0) \geq \max \left\{\beta \lambda \max \left\{E_{w}[\max \{w, 0\}], \max _{z} p(z) z\right\}-c, 0\right\}+\rho=$ $\max \{\beta \lambda K(0)-c, 0\}+\rho=\beta \lambda K(0)-c>0$ from (4) due to the assumptions that $\beta \lambda K(0)>c$ and $\rho=0$. Therefore, $V(0)>0$. If $Q_{0} \leq 0$ in (14), then $V(0)=\rho /(1-\beta)=0$ (note $\beta<1$ ), which contradicts $V(0)>0$. Hence, $Q_{0}>0$. This and Theorem 3 give the stated result.

(b) From (16) we have $h_{0}=\gamma\left(Q_{0}-Q_{1}\right)=\gamma \beta(1-$ q) $\lambda\left(K\left(h_{0}\right)-K\left(h_{1}\right)\right)$ due to (a). If $h_{0}=0$, then $K\left(h_{1}\right)=K\left(h_{0}\right)=$ $K(0)$; thus $h_{0}=h_{1}=0$. And if $h_{0}>0$, then $K\left(h_{0}\right)>K\left(h_{1}\right)$; thus $h_{0}<h_{1}$ due to Lemma A.1(b). Applying Theorem 2 on this completes the proof.

An implication of the result (a) is that when $\lambda \beta K(0)>$ $c$ and no profit is obtained from a sideline, it is optimal to search for customers in all states. The result (b) means that as $i$ increases, the decision maker will become more selective whether to choose the admission control policy or the pricing control policy.

\section{Numerical Studies}

In this section, we demonstrate cases where the switching occurs between the admission and the pricing control policies and examine the optimal search policies through some numerical experiments. The experiments have been made 


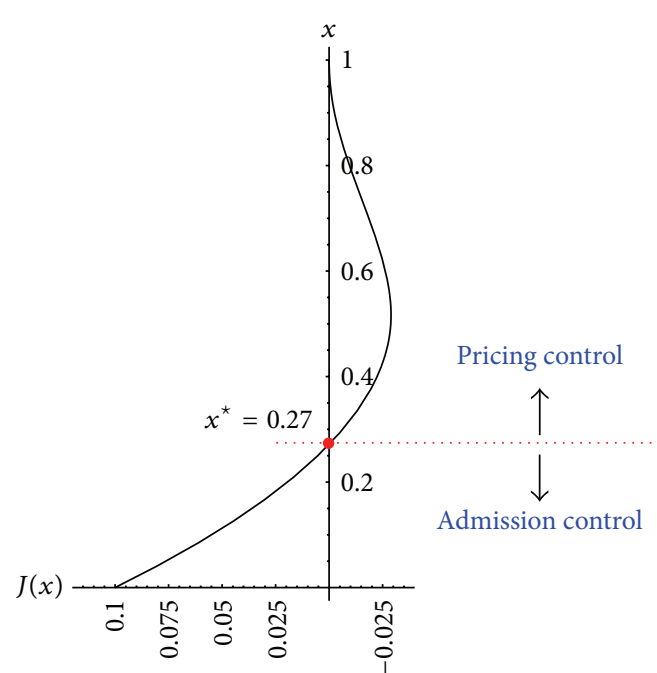

(a) $J(x)$-function

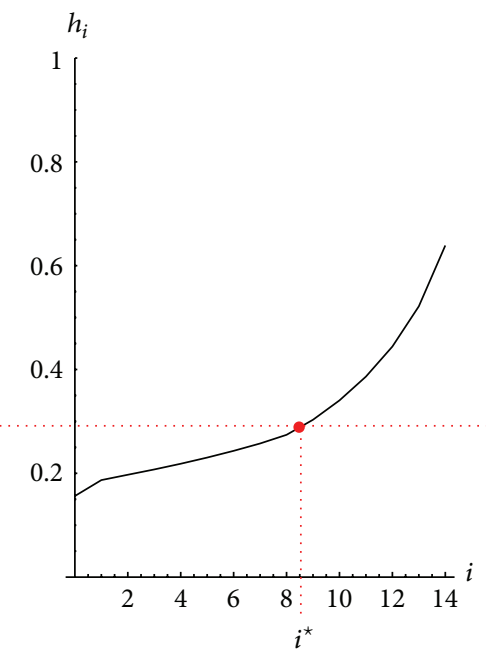

(b) Graph of $h_{i}$ when $\rho=0.0$

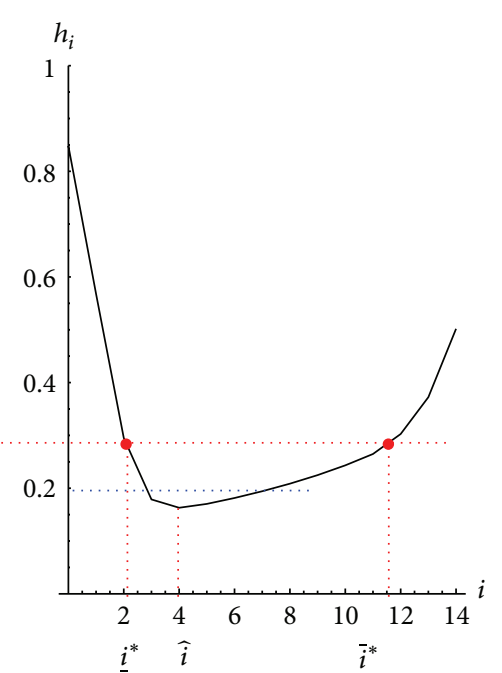

(c) Graph of $h_{i}$ when $\rho=0.3$

FIGURE 1: Graphs of $J(x)$ and $h_{i}$. If $J\left(h_{i}\right)>0$, adopting the admission control policy is optimal; otherwise, the pricing control policy is optimal.

TABLE 1: Optimal search policies.

\begin{tabular}{|c|c|c|c|c|c|c|c|c|c|c|c|c|c|c|c|c|}
\hline $\bar{i}$ & 0 & 1 & 2 & 3 & 4 & 5 & 6 & 7 & 8 & 9 & 10 & 11 & 12 & 13 & 14 & 15 \\
\hline$\rho=0.0$ & $\mathrm{C}$ & C & C & C & $\mathrm{C}$ & C & $\mathrm{C}$ & $\mathrm{C}$ & C & C & $\mathrm{C}$ & $\mathrm{C}$ & $\mathrm{C}$ & C & $\mathrm{C}$ & K \\
\hline$\rho=0.4$ & $\mathrm{~K}$ & $\mathrm{~K}$ & $\mathrm{~K}$ & $\mathrm{~K}$ & K & K & C & C & C & $\mathrm{C}$ & C & C & C & C & C & K \\
\hline
\end{tabular}

under the following conditions: $\beta=0.97, \lambda=0.99, c=0.05$, and $k=15$, and $F_{\xi}(x)$ and $F_{\alpha}(x)$ are the uniform distributions on $[0,1]$ and $[0.5,0.9]$, respectively. Note that $\beta \lambda K(0)-c \cong$ $0.29>0$ in this case.

5.1. Optimal Switching Strategy. In Figure 1, we present the graphs of $J(x)$ and $h_{i}$, where if $J\left(h_{i}\right)>0$, adopting the admission control policy is optimal; otherwise, the pricing control policy is optimal. The graph in Figure 1(a) depicts the function $J(x)$ on $[0,1]$, where $J(x)$ is less than zero and is increasing on $(0.89,1)$ (note $b^{o}=0.89$ ) by Lemma A.1(a). There exists $x^{\star}=0.27$ a solution of $J(x)=0$, such that $J(x)>0$ if $x<x^{\star}$, and $J(x) \leq 0$ otherwise.

Figure 1(b) demonstrates the fact that when $\rho=0, h_{i}$ indeed increases in $i$ as expected from Theorem 4(b). This creates a switching threshold $i^{\star}(=9)$ such that if $i<i^{\star}$, since $h_{i}<x^{\star}$, we have $J\left(h_{i}\right)>0$, and hence it is optimal to adopt the admission control policy. On the other hand, if $i \geq i^{\star}$, since $h_{i} \geq x^{\star}$, we get $J\left(h_{i}\right) \leq 0$; thus it is optimal to employ the pricing control policy. Therefore, when $i<i^{\star}\left(i \geq i^{\star}\right)$, since adopting the admission control (pricing control) policy is optimal, the optimal threshold $h_{i}$ (optimal pricing $z\left(h_{i}\right)$ ) should be set in such a way that it increases in the number of customers in the system. The implications of the monotonicity of $h_{i}$ and $z\left(h_{i}\right)$ are discussed in [18].

On the contrary, Figure 1(c) shows that when the sideline profit is sufficiently large as $\rho=0.3$, for example, $h_{i}$ is convex unimodal in $i$. In other words, there exists an $\widehat{i}(=4)$ such that if $i<\hat{i}$, then $h_{i}$ decreases, and if $i \geq \hat{i}$, then $h_{i}$ increases. Because of this unimodality, we discover the two switching thresholds $\underline{i}^{*}(=2)$ and $\bar{i}^{*}(=11)$ with respect to $i$, the number of customers in the system. Therefore, if $i \leq i^{*}$, since $h_{i} \geq x^{*}$, we have $J\left(h_{i}\right) \leq 0$, and hence it is optimal to employ the pricing control policy. When $i^{*}<i<\bar{i}^{*}$, then $h_{i}<x^{*}$, which gives $J\left(h_{i}\right)>0$; thus it is optimal to employ the admission control policy. Yet on $i \geq \bar{i}^{*}$, we note that it is again optimal to employ the pricing control policy. These facts create the following admission and pricing policies. When $i \leq \underline{i}^{*}$ and $\bar{i}^{*} \leq i$, the pricing control policy will be chosen, and hence the optimal pricing $z\left(h_{i}\right)$ should be set, respectively, to be decreasing and increasing in $i$. The movement of $z\left(h_{i}\right)$ in case of $i \leq i^{*}$ has arisen to obtain sufficiently large sideline profit by rejecting an arriving first-type customer. Now, when $\underline{i}^{*}<i<\bar{i}^{*}$, the admission policy should be chosen, and as a by-product the optimal admission threshold $h_{i}$ is convex unimodal in $i$. The convex unimodality creates the following DRV- (double critical value-) property. Assume that an arriving customer proposes the price $w=0.19$. In this case if $i \leq 3$, then $w>h_{i}$; therefore admitting the customer is optimal; if $3<i \leq 6$, then $w \leq h_{i}$; thus rejecting is optimal; and in neither of the above cases, since $w>h_{i}$, admission again becomes optimal. The values $i_{1}=3$ and $i_{2}=6$ will be the two critical values in this example.

5.2. Optimal Search Strategy. Table 1 represents the optimal search policies for a given $\rho$ in state $i(0,1, \ldots, 15)$. As proven in Theorem 4(a), when $\rho=0.0$, it is always optimal to conduct the search for customers in all states except the state $i=$ $k$, where the system capacity is full. In state $i=k$, both $\mathrm{C}$ and $\mathrm{K}$ can be optimal even though it appears in the table to 
skip the search. When the sideline profit is large enough as $\rho=0.4$, there exists an $i^{*}(=6)$ such that if $i<i^{*}$, it is optimal to skip, and otherwise to continue the search. This result reflects the fact that it is profitable to obtain a large sideline profit like in this case by skipping the search for the first-type customers. Therefore, it can be inferred that, as the sideline profit gets larger, the number of state changes from continuing to skipping in the optimal search policies would increase.

\section{Concluding Remarks}

We proposed a switching model between the admission and pricing control policies in relation to the sideline profits as well as the search costs. In this research we clarified the structural properties of the optimal switching strategies and the optimal search policies. According to our results, the optimal switching strategy depends on the shape of the function $J(x)$, and the optimal switching threshold exists in terms of the number of customers in the system under a certain condition. In particular, when the sideline profit is sufficiently large, switching between the policies has happened twice in two different states $i_{1}$ and $i_{2}\left(>i_{1}\right)$ in terms of the number of customers. This fact gives the following considerations. Let the number of customers, $i$, be sufficiently large as $i>i_{2}$. Then in order to avoid the system being full, the decision maker should reject customers with low price by setting the offering price high in pricing control. As a result, the number of customers in the system gradually decreases and reaches the switching threshold $i_{2}$, where the decision maker should switch the policy into the admission control policy. Now, let the number of customers, $i$, become sufficiently small. Then in order to obtain the sideline profit by rejecting low price of offers, the decision maker should set the admission threshold high. As a result, the number of customers gradually decreases and reaches the switching threshold $i_{1}$, where the decision maker should switch back to the pricing control policy.

The optimal search policies that were not introduced in [19] were also demonstrated here. If the search cost is sufficiently large, it is optimal to skip the search. However, when the search cost is sufficiently small, is it always optimal to continue the search? We showed that the answer is no! Even though the search cost is sufficiently small, yet the sideline profit is sufficiently large, it is optimal to skip the search as shown in Section 5.

As a general framework for the derivation of monotonicity properties, Koole [25] proposes a unified treatment of the various queueing models by concentrating on system events and the form of the value function instead of focusing on the value function itself. To investigate in what extent our model with the notion of search skipping fit within the Koole's framework would be an interesting topic for a further research.

Moreover, one could incorporate the following conditions which would make our model more practical: (1) future availability of once rejected customers, (2) customer's reneging from the queue, and (3) strategic interaction between the customer and the decision maker by introducing game theory and further investigate them.

\section{Appendix}

\section{Some Lemmas}

Here we arrange some lemmas that are used in the proofs of the theorems. We start with the lemma that represents the properties of the functions of $K(x)$ and $J(x)$, both of which play an important role in clarifying the characteristics of the optimal policies.

Lemma A.1. (a) $J(x)=0$ on $[b, \infty)$, and if $b^{o}<b$, then $J(x)$ is negative and increasing on $\left(b^{o}, b\right)$ (the properties of $J(x)$ function were proven in [19]. We however introduce the proofs here because of linguistic inaccessibility to the paper.).

(b) $K(x)$ is convex and decreasing on $(-\infty, \infty)$ and strictly decreasing on $(-\infty, b)$.

(c) $K(x)>0$ on $(-\infty, b)$ and $K(x)=0$ on $[b, \infty)$.

(d) $x+\nu K(x)$ is increasing on $(-\infty, \infty)$, where $0 \leq v \leq 1$.

Proof. (a) From (3) and the definition of $T_{w}(x)$ given in (8), we have $T_{w}(x)=\int_{0}^{\infty} \max \{w-x, 0\} E_{\alpha}\left[(1 / \alpha) f_{\xi}(w / \alpha)\right] d w$. Substituting $w=\alpha \xi$ into this equation leads to

$$
\begin{aligned}
T_{w}(x) & =E_{\alpha}\left[\alpha \int_{0}^{\infty} \max \left\{\xi-\frac{x}{\alpha}, 0\right\} f_{\xi}(\xi) d \xi\right] \\
& =E_{\alpha}\left[\alpha T_{\xi}\left(\frac{x}{\alpha}\right)\right]
\end{aligned}
$$

where $T_{\xi}(x)=E_{\xi}[\max \{\xi-x, 0\}]$. Here we note that if $b \leq x$, then $b \leq x / \alpha$ due to the inequality $0<\alpha \leq 1$. Subsequently, $T_{\xi}(x / \alpha)=0$ from (1). Hence from (A.1) we have $T_{w}(b)=$ $E_{\alpha}\left[\alpha T_{\xi}(b / \alpha)\right]=0$. Furthermore, if $b \leq x$, we have $z(x)=b$ maximizing $p(z)(z-b)$; hence $T_{p}(x)=\max p(z)(z-x)=$ $p(b)(b-x)=0$ because $p(b)=0$ from (1). Therefore we get $J(x)=0$ on $(b, \infty)$ from (9). Now, from the definition of $b^{o}$ in (11) and the fact that $T_{w}(x)$ is decreasing in $x$, we see that if $x<b^{o}$, then $T_{w}(x)>0$ and $T_{w}(x)=0$ otherwise. This validates the relation $b^{o} \leq b$. Suppose that $b^{o} \leq x<b$, then $T_{w}(x)=0$ as shown above and $T_{p}(x)>0$ due to (7) and (1). Thus, from (9) we have $J(x)=-T_{p}(x)<0$, which is increasing on $\left(b^{o}, b\right)$ because $T_{p}(x)$ is decreasing on $(-\infty, \infty)$ from (7).

(b) This is immediate from (10) and the fact that both $T_{p}(x)$ and $T_{w}(x)$ are convex and decreasing on $(-\infty, \infty)$ from (7) and (8).

(c) The proof follows from (a) (10) and the fact that $T_{p}(x)>0$ for $x<b$ and $T_{p}(x)=0$ for $b \leq x$, as shown in the proof of (a).

(d) We note that $x+v K(x)=\max \left\{x+\nu T_{w}(x), x+v T_{p}(x)\right\}$. Accordingly, we can prove the assertion by showing that each 
of $x+v T_{w}(x)$ and $x+v T_{p}(x)$ are increasing in $x$. Let $x_{1}<x_{2}$. Since $T_{w}(x)=E_{\alpha}\left[\alpha T_{\xi}(x / \alpha)\right]$ from (A.1), we have

$$
\begin{aligned}
& v T_{w}\left(x_{2}\right)+x_{2}-v T_{w}\left(x_{1}\right)-x_{1} \\
& =\left(x_{2}-x_{1}\right)+\nu E_{\alpha}\left[\alpha\left(T_{\xi}\left(\frac{x_{2}}{\alpha}\right)-T_{\xi}\left(\frac{x_{1}}{\alpha}\right)\right)\right] \\
& =\left(x_{2}-x_{1}\right)+v E_{\alpha} \\
& \cdot\left[\alpha \left(\int_{0}^{\infty} \max \left\{\xi-\frac{x_{2}}{\alpha}, 0\right\} d F(\xi)\right.\right. \\
& \left.\left.-\int_{0}^{\infty} \max \left\{\xi-\frac{x_{1}}{\alpha}, 0\right\} d F(\xi)\right)\right] \\
& \geq\left(x_{2}-x_{1}\right)+v E_{\alpha} \\
& \cdot\left[\alpha \left(\int_{x_{1} / \alpha}^{\infty}\left(\xi-\frac{x_{2}}{\alpha}\right) d F(\xi)\right.\right. \\
& \left.\left.-\int_{x_{1} / \alpha}^{\infty}\left(\xi-\frac{x_{1}}{\alpha}\right) d F(\xi)\right)\right] \\
& =\left(x_{2}-x_{1}\right)-v E_{\alpha} \\
& \cdot\left[\alpha\left(\frac{x_{2}}{\alpha}-\frac{x_{1}}{\alpha}\right)\left(1-F_{\xi}\left(\frac{x_{1}}{\alpha}\right)\right)\right] \\
& =\left(x_{2}-x_{1}\right)(1-v) E_{\alpha}\left[1-F_{\xi}\left(\frac{x_{1}}{\alpha}\right)\right] \geq 0,
\end{aligned}
$$

and so $T_{w}\left(x_{1}\right)+x_{1} \leq T_{w}\left(x_{2}\right)+x_{2}$. Thus $T_{w}(x)+x$ is increasing on $(-\infty, \infty)$. The proof for $T_{p}(x)+x$ can be found in [24].

The lemma given below guarantees the property of nonnegativity of $h_{i}$.

Lemma A.2. $h_{i} \geq 0$ for $i(0 \leq i \leq k)$.

Proof. Since $h_{i}$ is given by (6), to prove that the assertion is true we will show that $V(i)$ is decreasing in all $i$. Consider a value iteration algorithm corresponding to (4) and (5) for $t \geq$ 1 with $V_{0}(i)=0$ for all $i$. Clearly, $V_{0}(i)$ is decreasing in $i$. If we assume that $V_{t-1}(i)$ is decreasing in $i$, then it is immediate that $V_{t}(i)$ is decreasing in $i$ as well; hence the assertion holds.

Lemma A.3. If $Q_{i} \leq 0$ for a given $i(1 \leq i<k)$, then $h_{i-1} \geq h_{i}$.

Proof. Let $Q_{i} \leq 0$ for a given $i(1 \leq i<k)$. Then from (15) we have $V(i)=\gamma q \beta V(i-1)$, and hence $V(i+1)=\gamma q \beta V(i)+$ $\gamma \max \left\{Q_{i+1}, 0\right\}=(\gamma q \beta)^{2} V(i-1)+\gamma \max \left\{Q_{i+1}, 0\right\}$. Accordingly, we get

$$
\begin{aligned}
h_{i}-h_{i-1}= & 2 V(i)-V(i-1)-V(i+1) \\
= & 2 \gamma q \beta V(i-1)-V(i-1)-(\gamma q \beta)^{2} V(i-1) \\
& -\gamma \max \left\{Q_{i+1}, 0\right\} \\
= & -(1-\gamma q \beta)^{2} V(i-1)-\gamma \max \left\{Q_{i+1}, 0\right\} \leq 0
\end{aligned}
$$

due to $V(i-1) \geq 0$ from (14) and (15) and the fact that $1>\gamma q \beta$. Therefore $h_{i-1} \geq h_{i}$.

\section{Conflict of Interests}

The author declares that there is no conflict of interests regarding the publication of this paper.

\section{References}

[1] T. B. Crabill, D. Gross, and M. J. Magazine, "A classified bibliography of research on optimal design and control of queues," Operations Research, vol. 25, no. 2, pp. 219-232, 1977.

[2] L. A. DaSilva, "Pricing for QoS-enabled networks: a survey," IEEE Communications Surveys \& Tutorials, vol. 3, no. 2, pp. 2-8, 2000.

[3] M. Ghaderi and R. Boutaba, "Call admission control in mobile cellular networks: a comprehensive survey," Wireless Communications and Mobile Computing, vol. 6, no. 1, pp. 69-93, 2006.

[4] T. S. Kim, Y. Yang, J. C. Hou, and S. V. Krishnamurthy, "Resource allocation for QoS support in wireless mesh networks," IEEE Transactions on Wireless Communications, vol. 12, no. 5, pp. 2046-2054, 2013.

[5] W. Chung, S. Talluri, and R. Narasimhan, "Optimal pricing and inventory strategies with multiple price markdowns over time," European Journal of Operational Research, vol. 243, no. 1, pp. 130-141, 2015.

[6] J. Stidham and R. Weber, "A survey of markov decision models for control of networks of queues," Queueing Systems, vol. 13, no. 1-3, pp. 291-314, 1993.

[7] B. Kim and J. Kim, "Optimal admission control for two station tandem queues with loss," Operations Research Letters, vol. 42, no. 4, pp. 257-262, 2014.

[8] J. Mao and C. G. Cassandras, "Optimal admission control of discrete event systems with real-time constraints," Discrete Event Dynamic Systems: Theory and Applications, vol. 20, no. 1, pp. 37-62, 2010.

[9] J. Stidham, "Optimal control of admission to a queueing system," IEEE Transactions on Automatic Control, vol. 30, no. 8, pp. 705-713, 1985.

[10] L. Xia, "Event-based optimization of admission control in open queueing networks," Discrete Event Dynamic Systems: Theory and Applications, vol. 24, no. 2, pp. 133-151, 2014.

[11] T. Li, Y. Iraqi, and R. Boutaba, "Pricing and admission control for QoS-enabled Internet," Computer Networks, vol. 46, no. 1, pp. 87-110, 2004.

[12] O. Rubel, "Stochastic competitive entries and dynamic pricing," European Journal of Operational Research, vol. 231, no. 2, pp. 381-392, 2013.

[13] S. Transchel and S. Minner, "The impact of dynamic pricing on the economic order decision," European Journal of Operational Research, vol. 198, no. 3, pp. 773-789, 2009.

[14] P. S. You, "Dynamic pricing of inventory with cancellation demand," Journal of the Operational Research Society, vol. 54, no. 10, pp. 1093-1101, 2003.

[15] S. Yoon and M. E. Lewis, "Optimal pricing and admission control in a queueing system with periodically varying parameters," Queueing Systems. Theory and Applications, vol. 47, no. 3, pp. 177-199, 2004.

[16] N. Gans and S. Savin, "Pricing and capacity rationing in rentals," Working Paper, Wharton School of Business, University of Pennsylvania, 2005.

[17] S.-L. Hew and L. B. White, "Optimal integrated call admission control and dynamic pricing with handoffs and price-affected 
arrivals," in Proceedings of the Asia-Pacific Conference on Communications, pp. 396-400, October 2005.

[18] J.-D. Son, "Customer selection problem with profit from a sideline," European Journal of Operational Research, vol. 176, no. 2, pp. 1084-1102, 2007.

[19] J. D. Son, "Optimal switching strategy between admission control and pricing control in an $\mathrm{m} / \mathrm{m} / 1 / \mathrm{k}$ queueing system," Journal of the Korean Institute of Industrial Engineers, vol. 35, no. 2, pp. 161-167, 2009 (Korean).

[20] S. Ikuta, "Optimal stopping problem with uncertain recall," Journal of the Operations Research Society of Japan, vol. 31, no. 2, pp. 145-170, 1988.

[21] B.-K. Kang, "Optimal stopping problem with recall cost," European Journal of Operational Research, vol. 117, no. 2, pp. 222-238, 1999.

[22] T. Saito, "Optimal stopping problem with controlled recall," Probability in the Engineering and Informational Sciences, vol. 12, no. 1, pp. 91-108, 1998.

[23] S. Ross, Introduction to Stochastic Dynamic Programming, Academic Press, New York, NY, USA, 1983.

[24] S. Ikuta, "An integration of the optimal stopping problem and the optimal pricing problem-model with no recall," Discussion Paper No. 1084, Institute of Policy and Planning Sciences, University of Tsukuba, 2004.

[25] G. Koole, "Structural results for the control of queueing systems using event-based dynamic programming," Queueing Systems. Theory and Applications, vol. 30, no. 3-4, pp. 323-339, 1998. 


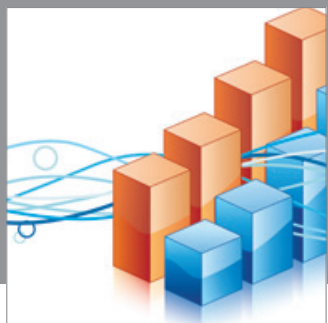

Advances in

Operations Research

mansans

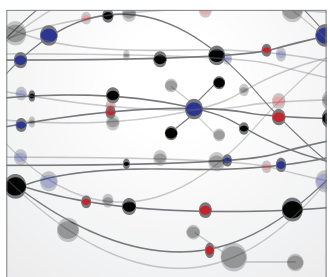

The Scientific World Journal
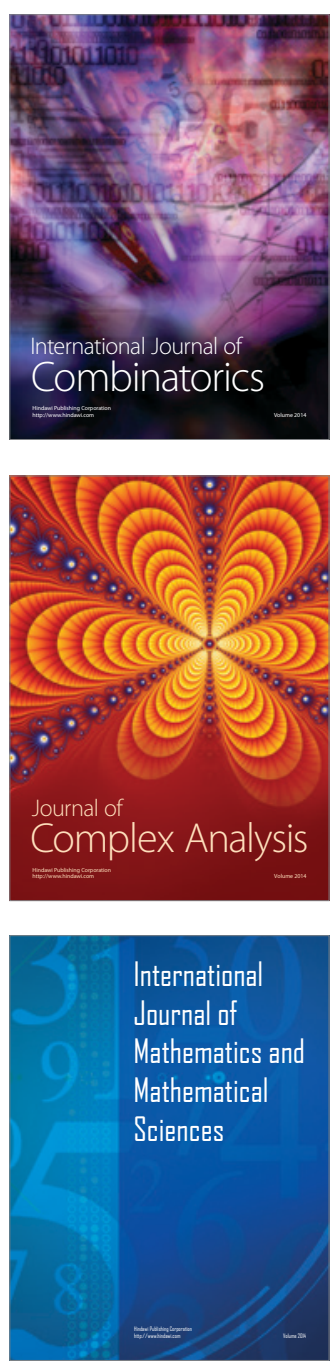
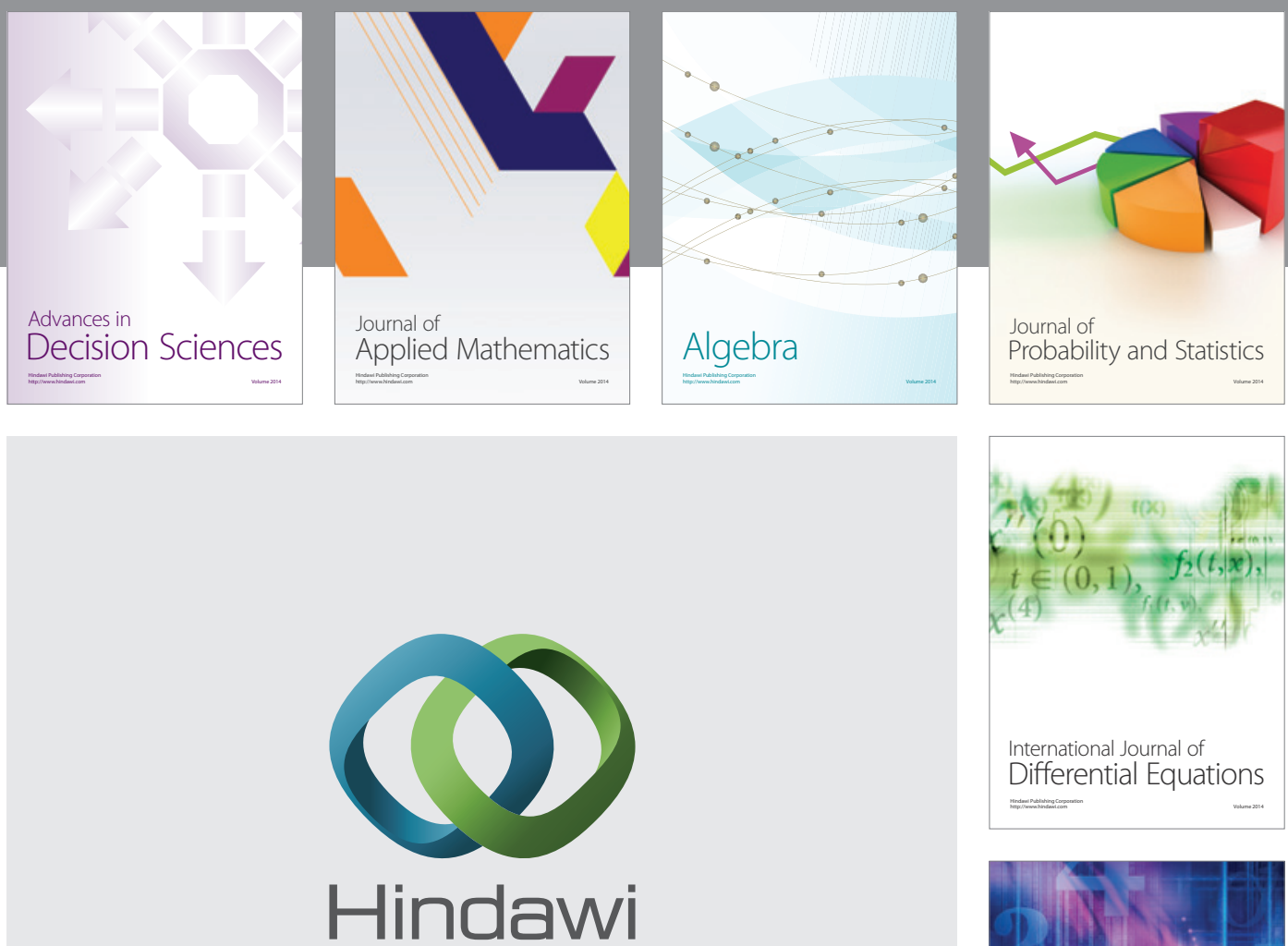

Submit your manuscripts at http://www.hindawi.com
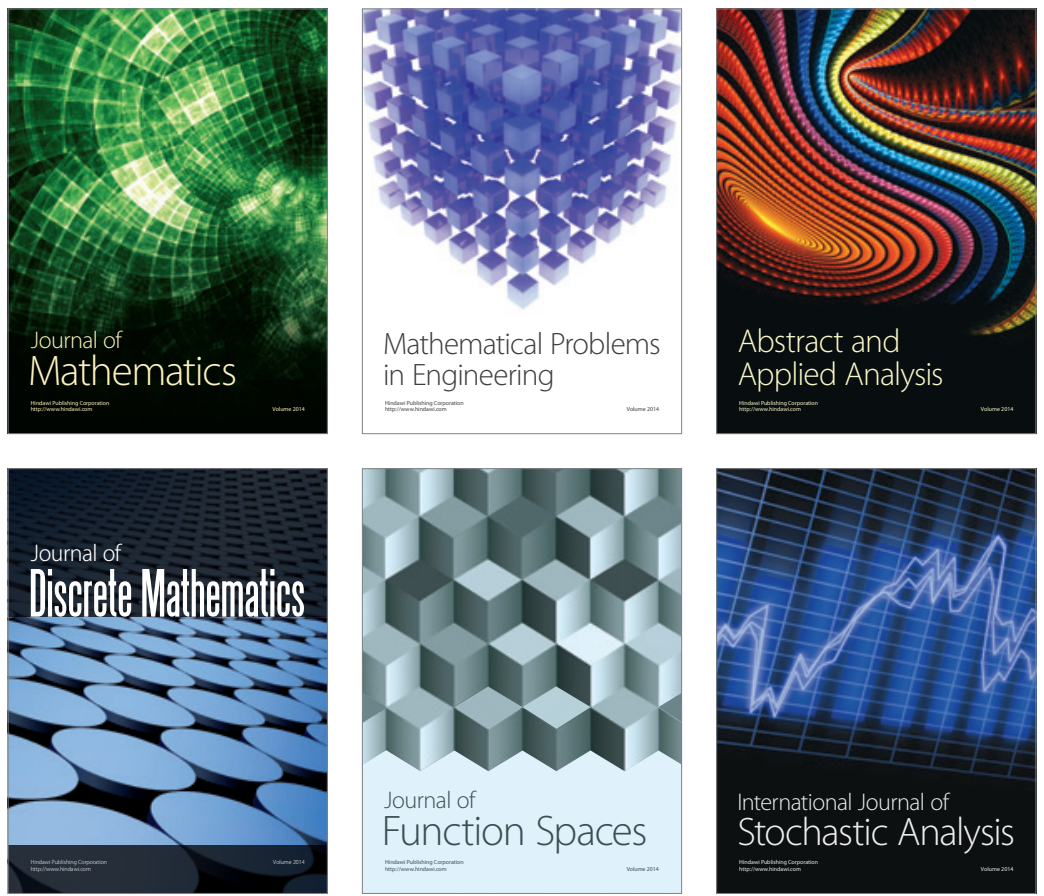

Journal of

Function Spaces

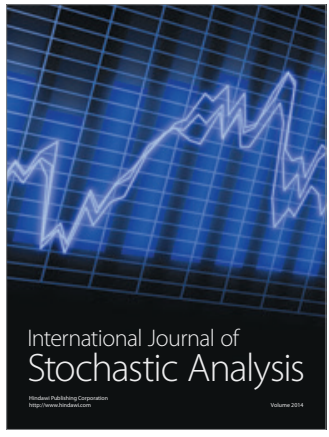

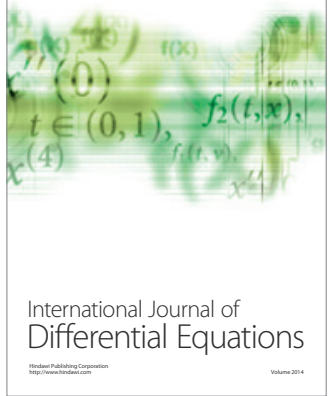
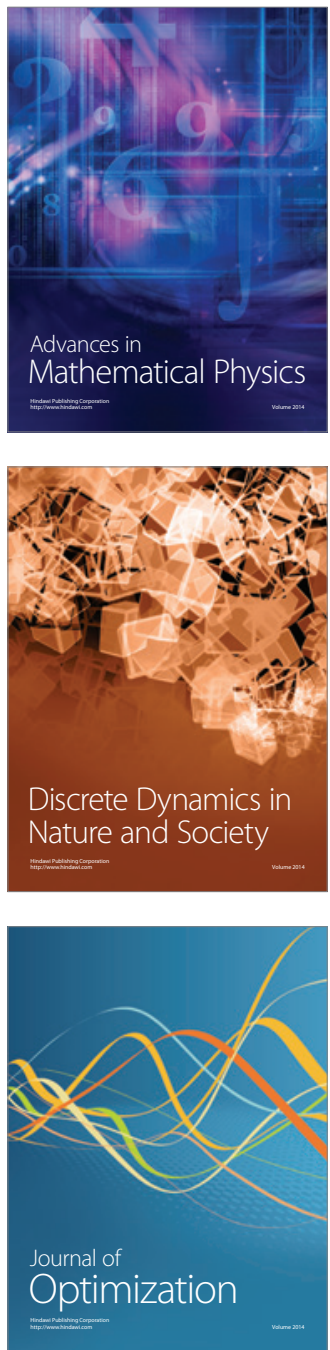